\title{
ASYMPTOTICALLY MONOTONE SOLUTIONS OF A NONLINEAR DIFFERENCE EQUATION
}

\author{
HORNG JAAN LI AND SUI SUN CHENG
}

\begin{abstract}
A bstract. Necessary conditions as well as sufficient conditions for the eventually positive solutions of a class of nonlinear difference equation to be monotone are derived.
\end{abstract}

\section{Introduction}

In this paper, we are concerned with a class of second order nonlinear difference equation of the form

$$
\Delta\left(p_{n-1} \Delta x_{n-1}\right)+q_{n} f\left(x_{n}\right)=0, \quad n=1,2,3, \cdots
$$

where $p_{n}>0$ for $n=0,1,2, \cdots$ and $f$ is a real nondecreasing function defined on $R$ such that sign $f(x)=\operatorname{sign} x$. Related works can be found in [1-7].

We shall be concerned with necessary conditions (see Section 3 ) as well as sufficient conditions (see Section 2) for the eventually positive solutions of (1) to be monotone. Such conditions are desirable since oscillation theorems for equation (1) can be derived if we impose these conditions (see Section 4).

A solution of (1) is a real sequence $x=\left\{x_{n}\right\}_{0}^{\infty}$ satisfying (1). It is clear that the standard existence and uniqueness theorem holds for (1). A nontrivial

Received May 5, 1990; revised March 5, 1993.

1991 Mathematics Subject Classification: 39A10.

Key words and phrases: Monotone solutions, nonlinear difference euqations, oscillation theorems. 
solution of (1) is said to be asymptotically or eventually positive if there exists an integer $N$ such that $x_{n}>0$ for $n \geq N$. It is said to be eventually increasing if there is some integer $M$ such that $\Delta x_{n}>0$ for $n \geq M$. Other concepts related to monotonicity of solutions or general sequences can be similarly defined.

A nontrivial real sequence $\left\{x_{n}\right\}_{0}^{\infty}$ is said to be oscillatory if for each $N \geq$ 0 , there exists an $n \geq N$ such that $x_{n} x_{n+1} \leq 0$, otherwise, $x$ is said to be nonoscillatory. Equation (1) is said to be oscillatory if all its nontrivial solutions are oscillatory. If $x_{m}=0$ for some $m \geq 1$, then $x_{m-1} \neq 0$ and $x_{m+1} \neq 0$ and $p_{m} x_{m+1}=-p_{m-1} x_{m-1}$ by (1), thus an oscillatory solution of (1) must change sign infinitely often, and a nonoscillatory solution is eventually positive or eventually negative.

The following lemma will be needed in the later discussions.

Lemma 1 . Let $f$ be a real nondecreasing function defined on $R$ such that $\operatorname{sign} f(x)=\operatorname{sign} x$. Let $\left\{x_{k}\right\}_{0}^{\infty}$ be a real sequence such that $x_{k}>0$ for $i \leq k \leq$ $j+1$. Then

$$
\sum_{k=i}^{j} \frac{\Delta x_{k}}{f\left(x_{k}\right)} \geq \int_{x_{i}}^{x_{j+1}} \frac{d u}{f(u)} \geq \sum_{k=i}^{j} \frac{\Delta x_{k}}{f\left(x_{k+1}\right)}
$$

Proof. For a fixed $k$ between $i$ and $j$, let

$$
g(t)=x_{k}+(t-k) \Delta x_{k}, \quad k \leq t \leq k+1
$$

then $g^{\prime}(t)=\Delta x_{k}$ and

$$
\frac{\Delta x_{k}}{f\left(x_{k}\right)} \geq \frac{g^{\prime}(t)}{f(g(t))} \geq \frac{\Delta x_{k}}{f\left(x_{k+1}\right)}, \quad k<t<k+1
$$

so that

$$
\frac{\Delta x_{k}}{f\left(x_{k}\right)} \geq \int_{k}^{k+1} \frac{g^{\prime}(t)}{f(g(t))}=\int_{x_{k}}^{x_{k+1}} \frac{d u}{f(u)} \geq \frac{\Delta x_{k}}{f\left(x_{k+1}\right)} .
$$

Thus

$$
\sum_{k=i}^{j} \frac{\Delta x_{k}}{f\left(x_{k}\right)} \geq \sum_{k=i}^{j} \int_{x_{k}}^{x_{k+1}} \frac{d u}{f(u)}=\int_{x_{i}}^{x_{j+1}} \frac{d u}{f(u)} \geq \sum_{k=i}^{j} \frac{\Delta x_{k}}{f\left(x_{k+1}\right)}
$$


as required.

\section{Sufficient Conditions}

In this section, we shall derive sufficient conditions for an eventually positive solution to be eventually monotone. For this purpose, let $x=\left\{x_{n}\right\}_{0}^{\infty}$ be an eventually positive solution of (1) such that $x_{n}>0$ for $n \geq N$. From (1), we have

$$
\frac{\Delta\left(p_{n-1} \Delta x_{n-1}\right)}{f\left(x_{n}\right)}+q_{n}=0, \quad n \geq N+1 \text {. }
$$

Summing (2) from $n=N+1$ to $n=k$, we have

$$
\frac{p_{k} \Delta x_{k}}{f\left(x_{k}\right)}-\sum_{n=N+1}^{k-1} p_{n} \Delta x_{n} \Delta\left\{\frac{1}{f\left(x_{n}\right)}\right\}-\frac{p_{N} \Delta x_{N}}{f\left(x_{N+1}\right)}+\sum_{n=N+1}^{k} q_{n}=0 .
$$

Since $f$ is nondecreasing,

$$
\Delta x_{n} \Delta\left\{\frac{1}{f\left(x_{n}\right)}\right\} \leq 0, \quad n \geq N+1
$$

so that

$$
\frac{p_{k} \Delta x_{k}}{f\left(x_{k}\right)} \leq \frac{p_{N} \Delta x_{N}}{f\left(x_{N+1}\right)}-\sum_{n=N+1}^{k} q_{n}
$$

or

$$
\frac{\Delta x_{k}}{f\left(x_{k}\right)} \leq \frac{p_{N} \Delta x_{N}}{p_{k} f\left(x_{N+1}\right)}-\sum_{n=N+1}^{k} \frac{q_{n}}{p_{k}} .
$$

Summing (4) from $k=j$ to $k=m$, we have, in view of Lemma 1, that

$$
\int_{x_{j}}^{x_{m+1}} \frac{d u}{f(u)} d t \leq \frac{p_{N} \Delta x_{N}}{f\left(x_{N+1}\right)} \sum_{k=j}^{m} \frac{1}{p_{k}}-\sum_{k=j}^{m} \sum_{n=N+1}^{k} \frac{q_{n}}{p_{k}} .
$$

Theorem. 1. Suppose the following conditions hold:

$$
\sum_{n=1}^{\infty} p_{n}^{-1}=\infty
$$




$$
\liminf _{m \rightarrow \infty} \sum_{n=k}^{m} q_{n}>0 \text { for all large } k .
$$

Then any eventually positive solution $\left\{x_{n}\right\}$ of $(1)$ is eventually increasing.

Proof. We may assume without loss of generality that $x_{n}>0$ for $n \geq N$ and

$$
\liminf _{m \rightarrow \infty} \sum_{n=N+1}^{m} q_{n}>0 .
$$

Suppose to the contrary that $\left\{\Delta x_{n}\right\}$ is not eventually positive, we may assume without loss of generality that $\Delta x_{N} \leq 0$. Then in view of (3) and the above inequality, we have $\Delta x_{n}<0$ for $n \geq N$, then, from (3) and (7), there is some integer $T$ such that

$$
p_{T} \Delta x_{T} \leq\left\{\frac{p_{N} \Delta x_{N}}{f\left(x_{N+1}\right)}-Q_{T}\right\} f\left(x_{T}\right)
$$

where

$$
Q_{k}=\sum_{n=N+1}^{k} q_{n}>0 \text { for } k \geq T .
$$

We now sum (1) from $n=T+1$ to $n=k$ and obtain

$$
p_{k} \Delta x_{k}-p_{T} \Delta x_{T}+\sum_{n=T+1}^{k} q_{n} f\left(x_{n}\right)=0
$$

so that

$$
\begin{aligned}
& p_{k} \Delta x_{k}=p_{T} \Delta x_{T}-\sum_{n=T+1}^{k} q_{n} f\left(x_{n}\right)=p_{T} \Delta x_{T}-\sum_{n=T+1}^{k} f\left(x_{n}\right) \Delta Q_{n-1} \\
\leq & \left\{\frac{p_{N} \Delta x_{N}}{f\left(x_{N+1}\right)}-Q_{T}\right\} f\left(x_{T}\right)-\left\{f\left(x_{k}\right) Q_{k}-\sum_{n=T+1}^{k-1} Q_{n} \Delta f\left(x_{n}\right)-f\left(x_{T+1}\right) Q_{T}\right\} \\
\leq & \left\{\frac{p_{N} \Delta x_{N}}{f\left(x_{N+1}\right)} f\left(x_{T}\right)+Q_{T} \Delta f\left(x_{T}\right)+\sum_{n=T+1}^{k-1} Q_{n} \Delta f\left(x_{n}\right)\right\} \\
\leq & \frac{p_{N} \Delta x_{N}}{f\left(x_{N+1}\right)} f\left(x_{T}\right)
\end{aligned}
$$


since $\Delta x_{k}<0$ for $k \geq T$ implies $\Delta f\left(x_{k}\right)<0$ for $k \geq T$.

As a consequence, divide the above inequality by $p_{k}$ and sumining, we obtain

$$
x_{m+1}-x_{T+1}=\sum_{n=T+1}^{m} \Delta x_{k} \leq \frac{p_{N} \Delta x_{N}}{f\left(x_{N+1}\right)} f\left(x_{T}\right) \sum_{n=T+1}^{m} p_{k}^{-1} .
$$

But (6) implies $x_{m+1} \rightarrow-\infty$ as $m \rightarrow \infty$, which contradicts our assumption. This completes our proof.

Note that in the above Theorem, condition (7) may be replaced by the following condition

$$
\liminf _{m \rightarrow \infty} \sum_{n=k}^{m} q_{n} \geq 0 \quad \text { for all large } k
$$

if we conclude that any eventually positive solution $\left\{x_{n}\right\}$ of $(1)$ is eventually nondecreasing. The proof is only a slight modification of that given above and is omitted.

Other results can be derived along similar lines of reasoning. For instance.

Theorem 2. Suppose there is some integer $M$ such that for each $T \geq M$, there is $j>T$ satisfying

$$
\limsup _{m \rightarrow \infty} \sum_{k=j}^{m} \sum_{n=T+1}^{k} \frac{q_{n}}{p_{k}}=\infty .
$$

Then for any eventually positive solution $\left\{x_{n}\right\}$ of (1), $\left\{x_{n}\right\}$ is either eventually increasing or

$$
\liminf _{n \rightarrow \infty} x_{n}=0 .
$$

Proof. As in the proof of Lemma 1 , suppose $N$ is an integer and $\left\{x_{n}\right\}$ is a solution of (1) such that $x_{n}>0$ for $n \geq N$,

$$
\limsup _{m \rightarrow \infty} \sum_{k=j}^{m} \sum_{n=N+1}^{k} \frac{q_{n}}{p_{k}}=\infty \text { for some } j>N,
$$


and $\Delta x_{n} \leq 0$ for $n=N$. If $\liminf _{n \rightarrow \infty} x_{n} \neq 0$, there then exists $\alpha>0$ and an integer $T \geq N$ such that $x_{m+1} \geq \alpha>0$ for all $m \geq T$. From (5), we obtain

$$
\int_{x_{j}}^{\alpha} \frac{d u}{f(u)} \leq \int_{x_{j}}^{x_{m+1}} \frac{d u}{f(u)} \leq-\sum_{k=j}^{m} \sum_{n=N+1}^{k} \frac{q_{n}}{p_{k}} .
$$

But our assumption implies that the limit superior of the right hand side diverges to $-\infty$, we arrive at a contradiction.

Theorem 3. Suppose

$$
\liminf _{k \rightarrow \infty} \frac{1}{p_{k}} \sum_{n=T+1}^{k} q_{n}>0, \quad \text { for all large } T .
$$

Then for any eventually positive solution $\left\{x_{n}\right\}$ of $(1),\left\{x_{n}\right\}$ is eventually increasing or $\left\{x_{n}\right\}$ is eventually decreasing to 0 .

Proof. As in the proof of Theorem 1, suppose $N$ is an integer and $\left\{x_{n}\right\}$ is a solution of (1) such that $x_{n}>0$ for $x \geq N$,

$$
\liminf _{k \rightarrow \infty} \frac{1}{p_{k}} \sum_{n=N+1}^{k} q_{n}>0
$$

and $\Delta x_{N} \leq 0$. Without loss of generality, we may assume

$$
\frac{1}{p_{k}} \sum_{n=N+1}^{k} q_{n} \geq \alpha>0 \text { for } k \geq T \text {. }
$$

Then from (4), we obtain

$$
\Delta x_{k} \leq-f\left(x_{k}\right) \sum_{n=N+1}^{k} \frac{q_{n}}{p_{k}} \leq-\alpha f\left(x_{k}\right)<0, \quad k \geq T
$$

which implies $\left\{x_{n}\right\}$ is eventually decreasing. If $\lim x_{n} \neq 0$, then there is some $\beta>0$ such that $x_{n} \geq \beta$ for all large $n$. Assume without loss of generality that $x_{n} \geq \beta$ for $n \geq T$, then summing the above inequality from $T$ to $m$, we have

$$
\beta \leq x_{m+1} \leq x_{T}-(m+1-T) \alpha f(\beta) \rightarrow-\infty .
$$


This contradiction completes our proof.

Theroem 4. Suppose condition (6) holds. Suppose further that there is some integer $M$ such that for each $T \geq M$, we can find $j>T$ satisfying

$$
\underset{m \rightarrow \infty}{\limsup } \sum_{k=j}^{m} \sum_{n=T+1}^{k} \frac{q_{n}}{p_{k}}>0 \text {. }
$$

Then for any eventually positive solution $\left\{x_{n}\right\}$ of (1), $\left\{x_{n}\right\}$ is either eventually increasing or

$$
\liminf _{n \rightarrow \infty} x_{n}=0
$$

Proof. As in the proof of Theorem 1, suppose $N$ is an integer and $\left\{x_{n}\right\}$ is a solution of (1) such that $x_{n}>0$ for $n \geq N$,

$$
\limsup _{m \rightarrow \infty} \sum_{k=j}^{m} \sum_{n=N+1}^{k} \frac{q_{n}}{p_{k}}>0 \text { for some } j>N
$$

and $\Delta x_{N} \leq 0$. Then in view of (4), there is an integer $T \geq N$ such that $\Delta x_{T}<0$. If $x_{n} \geq \alpha>0$ for $n$ larger than or equal to $T$, then from (5),

$$
\int_{x_{j}}^{\alpha} \frac{d u}{f(u)} \leq \int_{x_{j}}^{x_{m+1}} \frac{d u}{f(u)} \leq \frac{p_{N} \Delta x_{N}}{f\left(x_{N+1}\right)} \sum_{k=j}^{m} \frac{1}{p_{k}}-\sum_{k=j}^{m} \sum_{n=N+1}^{k} \frac{q_{n}}{p_{k}} .
$$

Since the limit superior of the right hand side is $-\infty$ by assumption, we arrive at a contradiction.

We remark that the condition (10) in the above Theorem can be replaced by

$$
\liminf _{m \rightarrow \infty} \sum_{k=j}^{m} \sum_{n=T+1}^{k} \frac{q_{n}}{p_{k}}>-\infty
$$

if we conclude that any eventually positive solution $\left\{x_{n}\right\}$ of (1) is either eventually nondecreasing or liminf $x_{n}=0$. Again the proof is omitted since it is a slight modification of that given above. 


\section{Necessary Conditions}

In this section, we shall consider necessary conditions for the eventually positive solutions of (1) to be monotone. To motivate the following discussions, we consider a very simple case. Assume (1) has an eventually positive increasing solution $\left\{x_{n}\right\}$ so that $x_{n}>0$ and $\Delta x_{n}>0$ for $n \geq N$. Then in view of (3), imposing the condition

$$
\limsup _{n \rightarrow \infty} \sum_{k=1}^{n} q_{k}=\infty
$$

would lead to a contradiction. In other words,

Theorem 5. If

$$
\limsup _{n \rightarrow \infty} \sum_{k=1}^{n} q_{k}=\infty
$$

then (1) cannot have an eventually positive nondecreasing solution.

More complicated necessary conditions can be derived along similar lines of reasoning. For this purpose, let $x$ be a nonoscillatory solution of (1) such that $x_{n}>0$ and $\Delta x_{n}>0$ for $n \geq N$. Further, let $\psi=\left\{\psi_{n}\right\}_{0}^{\infty}$ be a nonnegative sequence. If we multiply (1) by $\psi_{n} / p_{n} f\left(x_{n}\right)$ and then sum from $n=N+1$ to $n=k$, we obtain

$$
\sum_{n=N+1}^{k} \frac{\psi_{n}}{p_{n} f\left(x_{n}\right)} \Delta\left(p_{n-1} \Delta x_{n-1}\right)+\sum_{n=N+1}^{k} \frac{\psi_{n} q_{n}}{p_{n}}=0 .
$$

Summing the first term of the left hand side by parts, we obtain

$$
\begin{aligned}
& \frac{\psi_{k+1}}{p_{k+1} f\left(x_{k+1}\right)} p_{k} \Delta x_{k}-\frac{\psi_{N+1}}{p_{N+1} f\left(x_{N+1}\right)} p_{N} \Delta x_{N} \\
& -\sum_{n=N+1}^{k} p_{n} \Delta x_{n} \Delta\left\{\frac{\psi_{n}}{p_{n} f\left(x_{n}\right)}\right\}+\sum_{n=N+1}^{k} \frac{\psi_{n} q_{n}}{p_{n}}=0 .
\end{aligned}
$$

If we assume that $\left\{p_{n}\right\}$ is nondecreasing, then

$$
\Delta\left\{\frac{\psi_{n}}{p_{n} f\left(x_{n}\right)}\right\}=\left\{\frac{\psi_{n+1}}{p_{n+1} f\left(x_{n+1}\right)}-\frac{\psi_{n}}{p_{n} f\left(x_{n}\right)}\right\} \leq \frac{\Delta \psi_{n}}{p_{n+1} f\left(x_{n+1}\right)},
$$


and.

$$
p_{n} \Delta x_{n} \Delta\left\{\frac{\psi_{n}}{p_{n} f\left(x_{n}\right)}\right\} \leq p_{n} \Delta x_{n}\left\{\frac{\Delta \psi_{n}}{p_{n+1} f\left(x_{n+1}\right)}\right\} \leq \frac{\Delta x_{n} \Delta \psi_{n}}{f\left(x_{n+1}\right)}
$$

thus we have from (11) that

$$
\frac{\psi_{k+1} p_{k} \Delta x_{k}}{p_{k+1} f\left(x_{k+1}\right)}-\frac{\psi_{N+1} p_{N} \Delta x_{N}}{p_{N+1} f\left(x_{N+1}\right)}+\sum_{n=N+1}^{k} \frac{\psi_{n} q_{n}}{p_{n}} \leq \sum_{n=N+1}^{k} \frac{\Delta x_{n} \Delta \psi_{n}}{f\left(x_{n+1}\right)}
$$

Theorem 6. Suppose $\left\{p_{n}\right\}$ is nondecreasing and suppose there is a nonnegative and nonincreasing sequence $\psi$ such that

$$
\sum_{1}^{\infty} \frac{\psi_{n} q_{n}}{p_{n}}=+\infty
$$

then (1) cannot have an eventually positive nondecreasing solution.

Proof. Suppose (1) has a solution $x$ such that $x_{n}>0$ and $\Delta x_{n}>0$ for $n \geq N$. Then the left hand side of (12), in view of (13), is not bounded above. However, our assumptions on $\psi$ implies that the right hand side of (12) is nonpositive. A contradiction is thus obtained.

As an example, the following equation

$$
\Delta^{2} x_{n-1}+4 x_{n}^{\rho}=0, \quad n=1,2,3, \cdots
$$

where $\rho$ is the quotient of odd positive integers, cannot have an eventually positive increasing solution, since

$$
\sum_{1}^{\infty} \frac{q_{n}}{p_{n}}=+\infty
$$

Note also that it has an oscillatory solution $\left\{(-1)^{n}\right\}$.

The condition that $\psi$ is nonincreasing in the above Theorem can be replaced by other conditions. 
Theorem 7. Suppose $\left\{p_{n}\right\}$ is nondecreasing and suppose there is a nonnegative sequence $\psi=\left\{\psi_{n}\right\}$ such that $\left\{\Delta \psi_{n}\right\}$ is bounded and that (13) holds. Suppose further that

$$
0<\int_{\epsilon}^{\infty} \frac{d u}{f(u)}<\infty \quad \text { for some } \epsilon>0
$$

Then (1) cannot have an eventually positive nondecreasing solution.

Proof. Suppose (1) has a solution $x$ such that $x_{n}>0$ and $\Delta x_{n}>0$ for $n \geq N$. Then in view of Lemma 1 , we have

$$
\sum_{n=N+1}^{k} \frac{\Delta x_{n}}{f\left(x_{n+1}\right)} \leq \int_{x_{N+1}}^{x_{k+1}} \frac{d u}{f(u)}
$$

so that, in view of (14) and the boundedness of $\left\{\Delta \psi_{n}\right\}$,

$$
\sum_{n=N+1}^{\infty} \frac{\Delta x_{n} \Delta \psi_{n}}{f\left(x_{n+1}\right)} \leq \sum_{n=N+1}^{\infty} \frac{M \Delta x_{n}}{f\left(x_{n+1}\right)} \leq M \int_{x_{N+1}}^{\infty} \frac{d u}{f(u)}<\infty,
$$

where $\left|\Delta \psi_{n}\right| \leq M$ for $n=0,1,2, \cdots$. This implies that the left hand side of (12) is bounded above, which contradicts (13).

Note that in the proof of Theorem 7 , if $x$ is bounded by $Q$, then the first inequality in (16) is replaced by

$$
\sum_{n=N+1}^{\infty} \frac{\Delta x_{n} \Delta \psi_{n}}{f\left(x_{n+1}\right)} \leq M \int_{0}^{Q} \frac{d u}{f(u)}
$$

The following is now clear.

Theorem 8. Suppose $\left\{p_{n}\right\}$ is nondecreasng and suppose there is a nonnegative sequence $\psi=\left\{\psi_{n}\right\}$ such that $\left\{\Delta \psi_{n}\right\}$ is bounded and that (13) holds. Suppose further that

$$
0<\int_{0}^{\epsilon} \frac{d u}{f(u)}<\infty
$$

for some $\epsilon>0$. Then (1) cannot have a bounded solution which is eventually positive nondecreasing. 
Theorem 9. Suppose there is a nonnegative and nontrivial sequence $\psi=$ $\left\{\psi_{n}\right\}_{n=2}^{\infty}$ such that

$$
\limsup _{m \rightarrow \infty} \frac{\sum_{k=T}^{m} \sum_{n=T}^{k} \frac{\psi_{k+1} q_{n}}{p_{k}}}{\sum_{k=T}^{m} \frac{\psi_{k+1}}{p_{k}}}=\infty \text { for all large } T
$$

then (1) cannot have an eventually positive nondecreasing solution.

Proof. Suppose (1) has a nonoscillatory solution $x$ such that $x_{n} \geq 0$ and $\Delta x_{n}>0$ for $n \geq N$. If we multiply (4) by $\psi_{k+1}$, we get

$$
\frac{\psi_{k+1} \Delta x_{k}}{f\left(x_{k}\right)}+\sum_{n=N+1}^{k} \frac{\psi_{k+1} q_{n}}{p_{k}} \leq \frac{\psi_{k+1} p_{n} \Delta x_{N}}{f\left(x_{N+1}\right) p_{k}} .
$$

Thus

$$
\sum_{k=N+1}^{m} \sum_{n=N+1}^{k} \frac{\psi_{k+1} q_{n}}{p_{k}} \leq \frac{p_{N} \Delta x_{N}}{f\left(x_{N+1}\right)} \sum_{k=N+1}^{m} \frac{\psi_{k+1}}{p_{k}}
$$

which is a contradiction.

Theorem 10. Suppose

$$
\begin{gathered}
\sum_{k=1}^{\infty} q_{k}<\infty \\
\limsup _{m \rightarrow \infty} \sum_{n=T}^{m} \sum_{k=n+1}^{\infty} \frac{q_{k}}{p_{n}}=\infty \quad \text { for all large } T, \\
0<\int_{\epsilon}^{\infty} \frac{d u}{f(u)}<\infty \quad \text { for some } \epsilon>0 .
\end{gathered}
$$

Then equation (1) cannot have an eventually posititve nondecreasing solution.

Proof. Suppose to the contrary that $\left\{x_{n}\right\}$ is a solution of (1) such that $x_{n}>0$ and $\Delta x_{n}>0$ for $n \geq N$. Then in view of (3) and (18), we have

$$
\sum_{n=N+1}^{\infty} q_{n} \leq \frac{p_{N} \Delta x_{N}}{f\left(x_{N+1}\right)}
$$


From this inequality and (15), we then obtain

$$
\sum_{N=T}^{m} \sum_{n=N+1}^{\infty} \frac{q_{n}}{p_{N}} \leq \sum_{N=T}^{m} \frac{\Delta x_{N}}{f\left(x_{N+1}\right)} \leq \int_{x_{T}}^{x_{m}+1} \frac{d u}{f(u)} .
$$

In view of (19) and (14), a contradiction is clear.

Similar reasoning leads to the following

Theorem 11. Suppose

$$
0<\int_{0}^{\epsilon} \frac{d u}{f(u)}<\infty \quad \text { for some } \epsilon>0
$$

and that (18) and (19) hold. Then equation (1) cannot have a bounded solution which is eventually positive nondecreasing.

\section{Oscillation Theorems}

Each of the results derived in the last two sections has a dual statement valid for eventually negative decreasing solutions. This is clear from the fact that $\left\{x_{n}\right\}$ is a solution of (1) if and only if $\left\{-x_{n}\right\}$ is a solution of

$$
\Delta\left(p_{n-1} \Delta y_{n-1}\right)+q_{n} F\left(y_{n}\right)=0, \quad n=1,2,3, \cdots
$$

where $F(t)=-f(-t)$ for all $t$. Note that sign $F(t)=\operatorname{sign} t$ and $F$ is nondecreasing. As a consequence, each condition imposed on $\left\{p_{n}\right\}$ or $\left\{q_{n}\right\}$ in each of the previous result remains unchanged in the dual statement, while a condition imposed on the function $f$ has to be modified appropriately. As an example, a dual statement corresponding to Theorem 7 is as follows.

Theorem 12. Suppose $f$ is nondecreasing and suppose there is a nonnegative sequence $\left\{\psi_{n}\right\}$ such that $\left\{\Delta \psi_{n}\right\}$ is bounded and that (13) holds. Suppose further that

$$
0<\int_{-\epsilon}^{-\infty} \frac{d u}{f(u)}<\infty
$$


for some $\epsilon>0$. Then (1) cannot have an eventually negative nonincreasing solution.

Other results can be stated, but skipped because no new principle is involved.

The results derived in the previous two sections and their dual are useful in deriving oscillation theorems for equation (1). We illustrate the general procedure in a simple case. Let $\left\{x_{n}\right\}$ be a nonoscillatory solution of $(1)$, then as mentioned in Section 1, it is either eventually positive or eventually negative. Conditions (6) and (7) are sufficient for this solution to be eventually increasing in the former case and decreasing in the latter. If we now impose the condition in Theorem 5, we arrive at a contradiction.

Theorem 13. If the conditions imposed in Theorem 1 and Theroem 5 hold, then every solution of equation (1) is oscillatory.

Various combinations of the results in the previous two Sections and their dual will lead to oscillation theorems, but we shall confine ourself to an example. Let $r$ be a quotient of odd, positive integers, and let $\alpha$ be a real parameter. Let $p_{n}=1$ for $n=0,1,2, \cdots$ and

$$
q_{n}=(n+1)^{-\alpha r}\left\{(n+2)^{\alpha}+2(n+1)^{\alpha}+n^{\alpha}\right\}>0, \quad n=1,2,3, \cdots
$$

Then the equation

$$
\Delta^{2} x_{n-1}+q_{n} x_{n}^{r}=0, \quad n=1,2,3, \cdots
$$

has a soluton $x_{n}=(-1)^{n}(n+1)^{\alpha}$ for $n=0,1,2, \cdots$, which is oscillatory. Furthermore, we may make the following assertion: (a) If $\alpha<0$ and $r \geq(\alpha+1) / \alpha$, then $\sum q_{n}=\infty$, so that every nontrivial solution of (22) is oscillatory in view of Theorems 1,5 and their dual. (b) If $\alpha<0$ and $(\alpha+2) / \alpha \leq r<(\alpha+1) / \alpha$, then $r<1$ and $\sum(n+1) q_{n}=\infty$, so that every bounded nontrivial solution of (22) is oscillatory in view of Theorem 1,8 and their dual. (c) If $\alpha=0$, then $q_{n}=4$ for $n \geq 1$ and $\sum q_{n}=\infty$, so that every nontrivial solution of (22) is oscillatory 
in view of Theorems 1,5 and their dual. (d) If $\alpha>0$ and $r \leq(\alpha+1) / \alpha$, then $\sum q_{n}=\infty$, so that every nontrivial solution of (22) is oscillatory in view of Theorem 1,5 and their dual. (e) If $\alpha>0$ and $(\alpha+1) / \alpha<r \leq(\alpha+2) / r$, then $r>1$ and $\sum(n+1) q_{n}=1$, so that every nontrivial solution of $(22)$ is oscillatory in view of Theorems 1,7 and their dual.

\section{References}

[1] S. S. Cheng, "Sturmian comparison theorems for three-term recurrence equations", $J$. Math. Aral. Appl., 111 (1985), 465-474.

[2] S. S. Cheng, T. C. Yan and H. J. Li, "Oscillation criteria for second order difference equation", Funkcialaj Ekvacioj, 34 (1991), 223-239.

[3] J. W. Hooker and W. T. Patula, "Riccati type transformations for second-order linear difference equations", J. Math. Anal. Appl., 82 (1981), 451-462.

[4] J. W. Hooker and W. T. Patula, "A second order nonlinear difference equations: oscillation and asymptotic behavior", J. Math. Anal. Appl., 91 (1983), 9-29.

[5] Z. Nehari, "Oscillation criteria for second-order linear differential equations", Trans. AMS., 85 (1957), 428-445.

[6] B. Szmanda, "Oscillation theorems for nonlinear second order difference equations", $J$. Math. Ana3l. Appl., 79 (1981), 90-95.

[7] B. Szmanda, "Oscillation criteria for second order nonlinear difference equations", Annales Polonici Mathemtici, 43 (1983), 225-235.

Chienkuo Junior College of Technology, Taiwan, R. O. C.

Department of Mathematics, Tsing Hua University, Taiwan, R. O. C. 